\title{
Efeito da somatotropina bovina recombinante (rBST) sobre o desempenho produtivo e reprodutivo de vacas da raça Holandesa
}

[Effect of recombinant bovine somatotropin (rBST) on productive and reproductive performance of Holstein cows]

\author{
F.P. Rennó ${ }^{1}$, C.S. Lucci $^{1}$, A.G. Silva ${ }^{2}$, F.P. Rennó ${ }^{3}$, L.N. Rennó ${ }^{4}$, \\ B.P. Rennó Neto ${ }^{1}$, P.R. Cecon ${ }^{5}$, P.F. Barbosa ${ }^{2}$ \\ ${ }^{1}$ Faculdade de Medicina Veterinária e Zootecnia - USP \\ Av. Duque de Caxias Norte, 225 \\ 13635-900 - Pirassununga, SP \\ ${ }^{2}$ Embrapa Pecuária Sudeste - São Carlos, SP \\ ${ }^{3}$ UNIMONTES - Salinas, MG \\ ${ }^{4}$ UNIPAC - Juiz de Fora, MG \\ ${ }^{5}$ Deparmento de Informática - UFV - Viçosa, MG
}

\begin{abstract}
RESUMO
Avaliaram-se os efeitos da aplicação de rBST sobre o desempenho produtivo e reprodutivo de vacas da raça Holandesa no início da lactação. Foram avaliadas 41 vacas, dos 60 aos 150 dias de lactação, distribuídas em um delineamento inteiramente ao acaso, em três tratamentos: 1- sem aplicação de rBST (controle); 2- aplicação de rBST 60 dias após o parto (rBST-60); 3- aplicação de rBST 100 dias após o parto (rBST-100). A aplicação de rBST aumentou a produção de leite e a produção de leite corrigida a $4 \%$ de gordura em 14,0 e 13,2\%, respectivamente, quando administrada aos 60 dias pós-parto. Não se observou efeito da aplicação de rBST sobre a porcentagem e a produção de gordura do leite nem sobre o período de serviço, número de serviços por concepção e taxa de gestação aos 150 dias de lactação. $\mathrm{O}$ uso do rBST também não afetou o ganho de peso dos animais durante o período avaliado. A aplicação de rBST aos 60 dias de lactação proporcionou maiores pico de produção e persistência da lactação, sem, no entanto, influenciar o desempenho reprodutivo. A aplicação de rBST aos 100 dias de lactação não aumentou a produção de leite e o desempenho reprodutivo das vacas.
\end{abstract}

Palavras-chave: vaca leiteira, curva de lactação, produção de leite e gordura, reprodução, somatotropina

\begin{abstract}
The effects of recombinant bovine somatotropin (rBST) on production and reproductive parameters of Holstein dairy cows, from 60 to 150 days of lactation were evaluated. Forty-one cows were assigned to treatments: 1- no rBST application (control), 2- rBST application 60 days post calving (rBST-60), and 3rBST application 100 days after calving (rBST-100), in a completely randomized design. The rBST application 60 days post calving significantly increased milk production (14\%) and corrected milk production (13\%). No effects of rBST administration on milk fat production (\% and $\mathrm{kg} /$ day), days open, number of services/conception and pregnancy rate and on cow weight gain were observed. The rBST application 60 days after calving increased milk production peak and lactation persistence but did not change the reproductive performance. The rBST application at 100 days after calving did not increase milk production or the reproductive performance of cows.
\end{abstract}

Keywords: dairy cow, lactation curve, milk and fat yield, reproduction, somatotropin

Recebido em 10 de fevereiro de 2005

Aceito em 24 de outubro de 2005

E-mail: fprenno@hotmail.com 


\section{INTRODUÇÃO}

Uma das novas tecnologias que podem ser utilizadas para a obtenção de ganhos em produtividade em rebanhos leiteiros é a utilização da somatotropina recombinante bovina (rBST) para vacas em lactação. A resposta à utilização de rBST em vacas leiteiras se refere a aumentos de 10 a $15 \%$ na produção de leite (Etherton e Bauman, 1998)

Segundo Bauman (1992) e Metabolic... (1994), são possíveis acréscimos na produção de leite, após a aplicação da rBST, quando o manejo e os cuidados com os animais são excelentes. Níveis de resposta à aplicação de $\mathrm{rBST}$ de até $40 \%$ são citados por Peel et al. (1983), Richard et al. (1985) e Mattos (1998). Estudos envolvendo mais de 20.000 vacas mostraram que o rBST aumentou a produção de leite (Bauman, 1992).

O padrão de resposta à utilização da rBST é o aumento gradual da produção de leite poucos dias após a aplicação, sendo atingida a máxima resposta durante a primeira semana. Ao cessar a aplicação de rBST, gradualmente a produção de leite retorna aos níveis anteriores ao início da aplicação. Caso o tratamento seja continuado, o aumento na produção de leite é sustentado (Bauman et al., 1985). Dessa forma, a aplicação de rBST em vacas leiteiras influencia a produção de leite e o formato da curva de lactação (Bauman et al., 1999; Dunlap et al, 2000; LunaDominguez et al, 2000).

Em relação à composição do leite de vacas suplementadas com rBST, na maioria dos trabalhos, não se encontram alterações na porcentagem de gordura, proteína e lactose nos animais tratados (Barbano et al., 1992; Bauman, 1992; Bauman et al., 1999). Em função do aumento na produção de leite e da manutenção da porcentagem de sólidos no leite (gordura e proteína), a produção desses componentes é maior em vacas suplementadas com rBST (Etherton e Bauman, 1998). Em nove anos, foram avaliadas 27.000 vacas, em 340 rebanhos, e os dados demonstraram que a resposta ao rBST foi relativamente constante em cada ano, promovendo aumento de $894 \mathrm{~kg}$ de leite, $27 \mathrm{~kg}$ de gordura e $31 \mathrm{~kg}$ de proteína no leite por lactação, com o padrão de contagem de células somáticas não sendo influenciado (Bauman et al., 1999). A utilização da rBST também não influenciou as características importantes do leite para o processamento industrial (Eppard et al., 1985; Peel e Bauman, 1987; Barbano et al., 1992; Laurent et al., 1992).

Em relação aos efeitos sobre a reprodução, Mattos (1998) relatou que a utilização de rBST em vacas lactantes apresenta resultados discrepantes em relação ao desempenho reprodutivo. A utilização de $\mathrm{rBST}$ em vacas de alta produção tem influenciado o número de serviços por concepção, a taxa de gestação e o intervalo parto-concepção. Burton et al. (1990) relataram tendências de aumento no intervalo parto-concepção (5 a 30 dias) e no número de serviços por concepção $(0,5$ a 1$)$ e diminuição da taxa de gestação $(-5$ a $-10 \%)$ para animais tratados com rBST. Santos et al. (2000) citaram que vacas tratadas com rBST apresentaram maior número de serviços por concepção e maior intervalo parto-concepção do que animais nãotratados.

Luna-Dominguez et al. (2000) verificaram redução na taxa de gestação para animais tratados com rBST, sem observar influência da aplicação do rBST sobre o intervalo partoconcepção e o número de serviços por concepção. Cole et al. (1991) e Zhao et al. (1992) observaram não haver diferenças em relação à taxa de gestação, número de serviços por concepção e intervalo parto-concepção em vacas tratadas ou não com rBST. Lucy (2000) citou que a aplicação de rBST não apresentou efeito direto sobre o desempenho reprodutivo de vacas em lactação.

Na avaliação dos efeitos da aplicação de rBST na variação do peso corporal, Huber et al. (1997) e Tarazon-Herrera et al. (2000) verificaram que o peso corporal não foi afetado em vacas tratadas com rBST. Os autores comentaram que mudanças nessas variáveis são difíceis de serem identificadas em experimentos com rBST, pois elas dependem de muitos fatores, principalmente do balanço de energia.

Zhao et al. (1992) avaliaram a aplicação de rBST em vacas leiteiras a partir da quarta semana até 280 dias de lactação, sendo aplicado diariamente (10,3mg/dia) e a cada 14 dias (injeções de $350 \mathrm{mg}$ ), e verificaram médias de ganho de peso semelhantes entre esses tratamentos e os animais que não receberam $\operatorname{rBST}(490,430$ e $510 \mathrm{~g} / \mathrm{dia}$, 
respectivamente). Segundo Gibson et al. (1992), a administração de rBST, dos 28 aos 294 dias da lactação e em duas dosagens $(250$ e $500 \mathrm{mg}$ a cada 14 dias), não afetou o ganho de peso das vacas em relação aos animais-controle $(410,380$ e $360 \mathrm{~g} /$ dia, respectivamente).

O presente estudo teve o objetivo de avaliar os efeitos da aplicação de rBST, aos 60 e 100 dias pós-parto, sobre a produção de leite e gordura, a curva de lactação, o desempenho reprodutivo e a variação do peso corporal de vacas da raça Holandesa no início da lactação.

\section{MATERIAL E MÉTODOS}

O experimento foi conduzido no sistema de produção de leite do Centro de Pesquisa de Pecuária do Sudeste (Embrapa Pecuária Sudeste), em São Carlos, de abril a dezembro de 1999. Foram utilizadas 41 vacas em lactação da raça Holandesa, puras de origem (PO), com média de produção de leite de $30,0 \mathrm{~kg} / \mathrm{dia}$, avaliadas dos 60 aos 150 dias de lactação, distribuídas em um delineamento inteiramente ao acaso, sendo as vacas aleatoriamente alocadas conforme atingiam os 60 dias de lactação em um dos três tratamentos: I - sem aplicação de rBST (controle), 15 vacas; II - aplicação de rBST aos 60 dias após o parto (rBST-60), 15 vacas; e III aplicação de rBST aos 100 dias, após o parto (rBST-100), 11 vacas. A rBST $^{1}$ utilizada foi administrada de acordo com as recomendações do fabricante $(500 \mathrm{mg}$ de sometribove zinco a cada 14 dias).

As vacas foram mantidas estabuladas durante 30 dias antes do período experimental para adaptação ao manejo e verificação de possíveis patologias pós-parto. No tratamento III (rBST100), quatro vacas foram eliminadas do experimento antes da aplicação do rBST devido a problemas de saúde. Os animais foram alimentados duas vezes ao dia, na forma de dieta total, sendo a ração formulada segundo o NRC (Nutrient..., 1989), com base na matéria seca, contendo $50 \%$ de volumoso (silagem de milho) e $50 \%$ de alimentos concentrados (farelo de soja, milho triturado, uréia, minerais e vitaminas). As sobras foram retiradas dos cochos, uma vez por dia, pela manhã. A composição bromatológica da silagem de milho, a do concentrado e a da ração encontram-se na Tab. 1.

Tabela 1. Teores médios, em porcentagens, de matéria seca (MS), proteína bruta $(\mathrm{PB})$, proteína não degradável no rúmen (PNDR), extrato etéreo (EE), fibra em detergente neutro (FDN), fibra em detergente ácido (FDA), nutrientes digestíveis totais (NDT), cálcio (Ca) e fósforo (P), do concentrado, da silagem de milho e da ração para vacas da raça Holandesa

\begin{tabular}{lccc}
\hline Itens & Concentrado & Silagem de milho & Ração \\
\hline MS (\%) & 90,00 & 33,89 & 61,95 \\
$\mathrm{~PB}^{1}$ & 27,01 & 7,51 & 17,26 \\
$\mathrm{PNDR}^{23}$ & 41,07 & 31,00 & 36,04 \\
$\mathrm{EE}^{1}$ & 2,73 & 3,20 & 2,97 \\
$\mathrm{FDN}^{1}$ & 8,98 & 45,00 & 26,99 \\
$\mathrm{FDA}^{1}$ & 5,68 & 26,60 & 16,14 \\
$\mathrm{NDT}^{13}$ & 77,48 & 66,00 & 71,74 \\
$\mathrm{Ca}^{1}$ & 1,00 & 0,23 & 0,62 \\
$\mathrm{P}^{1}$ & 0,60 & 0,22 & 0,41 \\
\hline
\end{tabular}

${ }^{1}$ Porcentagem na MS

${ }^{2}$ Porcentagem da $\mathrm{PB}$

${ }^{3}$ Dados retirados do (Nutrient... 1989).

As análises da composição química da silagem de milho e do concentrado determinaram os teores de matéria seca (MS), extrato etéreo (EE), fibra em detergente neutro (FDN), fibra em detergente ácido (FDA) e proteína bruta (PB), conforme técnica descrita por Silva (1990).

As vacas foram ordenhadas mecanicamente, duas vezes ao dia, sendo o controle leiteiro realizado quinzenalmente. A cada 30 dias, foram colhidas amostras de leite das vacas nas duas ordenhas para posterior determinação de gordura. O leite foi corrigido para o teor de $4 \%$ de gordura (Nutrient... 1989; 2001).

Os animais foram pesados a cada 14 dias, pela manhã, antes do fornecimento da ração, levandose em consideração a ordem dos animais dentro dos tratamentos. As fêmeas que apresentaram partos normais foram submetidas a exame ginecológico para diagnóstico de possíveis alterações anatômicas ou patológicas, excluindose do experimento aquelas com problemas.

\footnotetext{
${ }^{1}$ Lactotropin ${ }^{\circledR}$ Injetável - Elanco Saúde Animal - São Paulo, SP.
} 
Os cios foram identificados durante o manejo das ordenhas, e as vacas inseminadas a partir do primeiro cio após 60 dias de parição, de forma que não havia vacas inseminadas ou gestantes no início do período experimental. Promoveu-se o diagnóstico de gestação após 45 dias da inseminação artificial, por meio de palpação retal, com confirmação aos 60 dias após a inseminação. Foram avaliadas a taxa de gestação, o intervalo parto-concepção e o número de serviços por concepção até 150 dias pós-parto.

O delineamento experimental utilizado foi o inteiramente ao acaso, com 15, 15 e 11 repetições, respectivamente, para os tratamentos controle, rBST-60 e rBST-100. Idade da vaca ao parto (em meses) e mês de parição foram incluídos no modelo como covariáveis. Quando essas covariáveis não foram significativas $(\mathrm{P}<0,05)$ para cada variável estudada, foram excluídas do modelo, sendo refeita a análise.

Realizaram-se análises de variância para as variáveis produção de leite, produção de leite corrigida a 4\% de gordura, produção de gordura ( $\%$ e $\mathrm{kg} / \mathrm{dia})$ e peso corporal. As médias foram comparadas utilizando-se o teste Tukey, adotando-se o nível de 5\% de significância. Na avaliação do peso corporal, optou-se pelo desdobramento da interação tratamento $\mathrm{x}$ dias em lactação, estudando-se a variável peso corporal em função dos dias para cada tratamento.

Com objetivo de estudar os efeitos dos tratamentos com rBST, por um período logo após sua aplicação, foi feita análise de regressão do peso corporal em função dos dias de lactação e, para avaliação da curva de lactação, foi feita uma análise de regressão da produção de leite em função dos dias de lactação.

Para as características reprodutivas, com exceção da taxa de gestação, foram realizados os testes de Bartllet e de Lilliefors para se verificar a homogeneidade e a normalidade dos dados, respectivamente. Para intervalo parto-concepção e número de inseminações por concepção, foram realizadas análises de variância. Para a taxa de gestação, foi aplicado o teste qui-quadrado.

Os dados foram analisados utilizando-se o programa SAEG (Sistema... 1999).

\section{RESULTADOS E DISCUSSÃO}

$\mathrm{Na}$ Tab. 2, são apresentadas as médias de produção de leite (PL), produção de leite corrigida para $4 \%$ de gordura (PLC), porcentagem de gordura $(\mathrm{G} \%)$ e produção de gordura $(\mathrm{Gkg})$ e seus respectivos coeficientes de variação. Foram observados efeitos $(\mathrm{P}<0,05)$ da administração de rBST sobre a produção de leite e produção de leite corrigida para $4 \%$ de gordura. As maiores PL e PLC foram observadas para o tratamento com aplicação de rBST aos 60 dias de lactação, que foram diferentes do tratamentocontrole, porém não diferiram do tratamento com rBST aos 100 dias. Este último não diferiu do grupo-controle.

Tabela 2. Médias e coeficientes de variação (CV) da produção de leite (PL), produção de leite corrigida (PLC) para 4\% de gordura, em $\mathrm{kg} / \mathrm{dia}$, e da produção de gordura, em porcentagem $(\mathrm{G} \%)$ e em kg/dia (Gkg), segundo os tratamentos

\begin{tabular}{lcccc}
\hline Tratamento & $\begin{array}{c}\text { PL } \\
(\mathrm{kg} / \mathrm{dia})\end{array}$ & $\begin{array}{c}\text { PLC } \\
(\mathrm{kg} / \mathrm{dia})\end{array}$ & $\begin{array}{c}\mathrm{G} \% \\
(\%)\end{array}$ & $\begin{array}{c}\mathrm{Gkg} \\
(\mathrm{kg} / \mathrm{dia})\end{array}$ \\
\hline Controle & $30,25 \mathrm{~b}$ & $27,23 \mathrm{~b}$ & $3,37 \mathrm{a}$ & $1,01 \mathrm{a}$ \\
rBST-60 & $34,50 \mathrm{a}$ & $30,82 \mathrm{a}$ & $3,29 \mathrm{a}$ & $1,14 \mathrm{a}$ \\
rBST-100 & $31,40 \mathrm{a} \mathrm{b}$ & $28,78 \mathrm{a} \mathrm{b}$ & $3,50 \mathrm{a}$ & $1,08 \mathrm{a}$ \\
CV $(\%)$ & 13,08 & 12,64 & 11,57 & 14,22 \\
\hline
\end{tabular}

$\mathrm{Na}$ coluna, médias seguidas de letras distintas diferem entre si $(\mathrm{P}<0,05)$, segundo o teste Tukey.

As vacas do tratamento rBST-60 aumentaram a PL em média $14,0 \%(4,25 \mathrm{~kg})$ e a PLC, $13,2 \%$ $(3,59 \mathrm{~kg})$, comparadas com as vacas do tratamento-controle. Comparando-se os animais do tratamento rBST-100 com os do grupocontrole, verificaram-se, em média, aumentos na PL de $3,8 \%(1,15 \mathrm{~kg})$ e na PLC de $5,7 \%(1,55 \mathrm{~kg})$. Ao se relacionar ambos os tratamentos com rBST, as vacas do tratamento rBST-60 aumentaram a PL em $9,9 \%(3,10 \mathrm{~kg})$ e a PLC em $7,1 \%(2,04 \mathrm{~kg})$ em relação às do tratamento rBST-100.

Os resultados são semelhantes aos relatados por Huber et al. (1997), utilizando a mesma dosagem de rBST no mesmo intervalo e dia de início de aplicação (rBST-60), em que os animais suplementados produziram $14 \%(3,7 \mathrm{~kg})$ a mais de leite por dia que os animais-controle. Assemelham-se também aos resultados obtidos por Lucci et al. (1998), Bauman et al. (1999), 
Dunlap et al. (2000) e Tarazon-Herrera et al. (2000).

$\mathrm{Na}$ Tab. 2, são apresentadas as médias da produção de gordura (\% e kg/dia), de acordo com os tratamentos até os 150 dias de lactação. Não se verificaram diferenças $(\mathrm{P}>0,05)$ nas porcentagens de gordura no leite. TarazonHerrera et al. (2000) verificaram que as porcentagens de gordura do leite não foram alteradas pelo tratamento com rBST, apresentando média de 3,53\%. Burton et al. (1990), Gibson et al. (1992) e Bauman et al. (1999) também não encontraram diferenças nas porcentagens de gordura do leite de vacas suplementadas ou não com rBST no início da lactação.

Os tratamentos com rBST aos 60 e 100 dias não resultaram em diferenças significativas na produção de gordura em relação ao tratamento- controle. Na avaliação de 340 rebanhos sem e com a suplementação com rBST (Bauman et al., 1999) foram obtidas médias de 0,96 e $1,18 \mathrm{~kg} / \mathrm{dia}$ de gordura, respectivamente.

Na Fig. 1, mostra-se o efeito da aplicação de rBST sobre a PL e PLC em função dos dias de lactação. Após a aplicação de rBST aos 60 dias de lactação, houve maior resposta no aumento da PL, resultando em maior persistência de lactação. Com relação ao tratamento $\mathrm{HBST}-100$, verifica-se estabilização seguida de diminuição menos acentuada na curva de lactação, em comparação ao tratamento-controle. $\mathrm{Na}$ Tab. 3, são apresentadas as equações de regressão com os respectivos coeficientes de determinação para a produção de leite nos primeiros 45 dias após a aplicação de rBST em cada tratamento (rBST-60 e rBST-100) e para o controle, nos respectivos períodos.
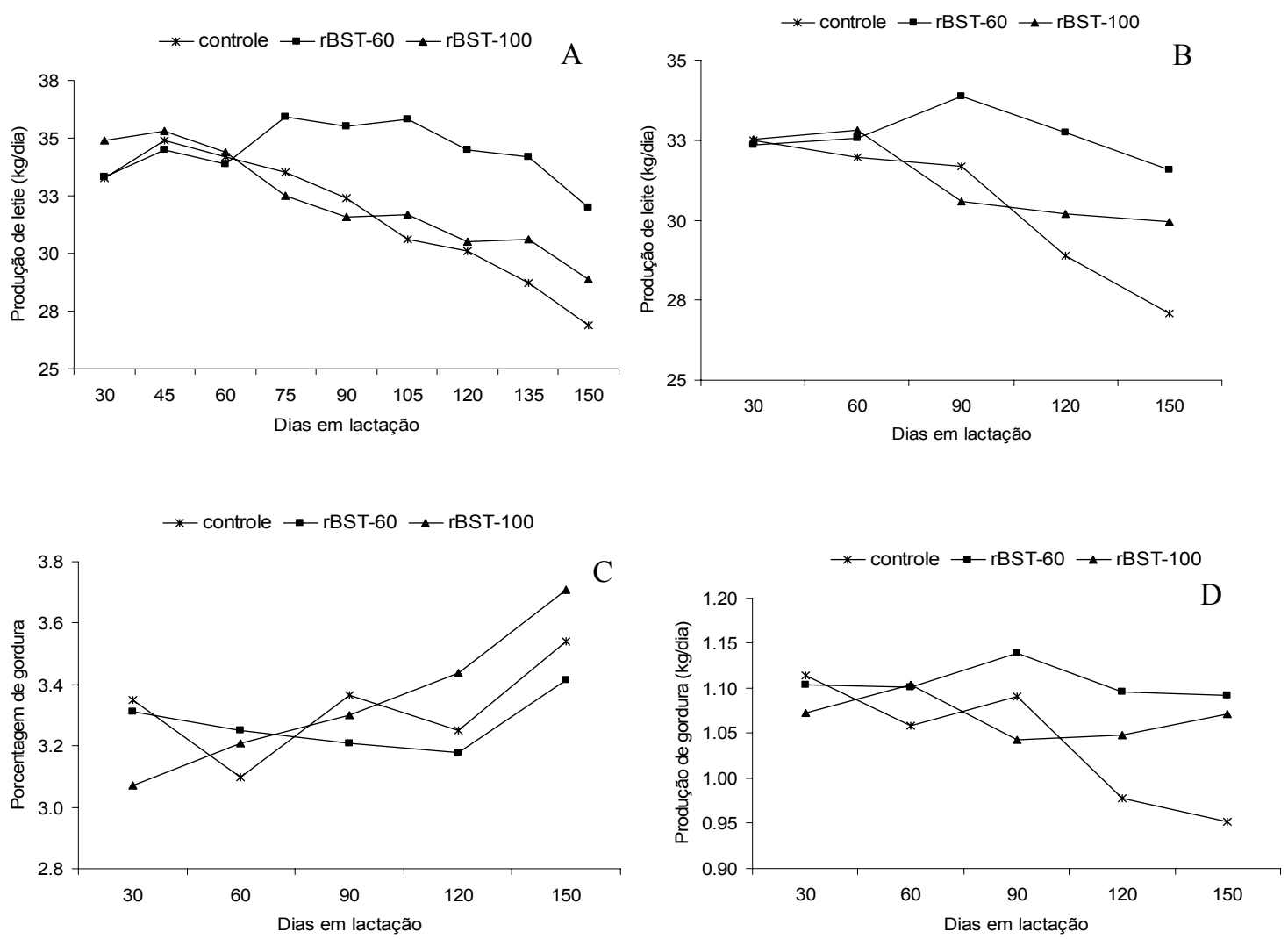

Figura 1. Produção de leite (A), produção de leite corrigida para 4\% de gordura (B), porcentagem (C) e produção de gordura do leite (D) em função dos dias de lactação, segundo os tratamentos, obtidas de vacas da raça Holandesa. 
Tabela 3. Equações de regressão (ER) e coeficientes de determinação $\left(\mathrm{R}^{2}\right)$ para produção de leite nos primeiros 45 dias após a aplicação de rBST, segundo os tratamentos e dias da lactação

\begin{tabular}{lclc}
\hline Tratamento & Dias & ER & $\mathrm{R}^{2}$ \\
\hline Controle & $75-120$ & $\hat{Y}=39,432-0,0809 \mathrm{D}$ & 0,97 \\
& $105-150$ & $\hat{Y}=39,624-0,0836 \mathrm{D}$ & 0,95 \\
rBST-60 & $75-120$ & $\hat{Y}=37,764-0,0257 \mathrm{D}$ & 0,62 \\
rBST-100 & $105-150$ & $\hat{Y}=37,938-0,0555 \mathrm{D}$ & 0,88 \\
\hline
\end{tabular}

Com o avançar da lactação, a PL (kg/dia) diminuiu linearmente em função dos dias de lactação (Tab. 3). Para o tratamento com rBST60 , entre 75 a 120 dias de lactação, a cada dia da lactação, a PL diminuiu $25,7 \mathrm{~g}$, enquanto para o mesmo período no tratamento-controle, a PL decresceu 80,9g/dia. Quando se avalia o tratamento com rBST-100, entre 105 a 150 dias de lactação, a cada dia da lactação, a PL diminuiu $55,5 \mathrm{~g}$; para o mesmo período no tratamento-controle, a PL foi reduzida a $83,6 \mathrm{~g} / \mathrm{dia}$. Esses resultados demonstram que a aplicação de rBST aumenta a persistência de lactação e afeta a curva de lactação de vacas leiteiras.

Ao ser comparada a magnitude da diminuição da PL dos tratamentos com rBST aos 60 e 100 dias, verifica-se maior persistência na PL com rBST aos 60 dias que aos 100 dias, com 25,7 e $55,5 \mathrm{~g} /$ dia de decréscimo, respectivamente (Fig. 1). Estes resultados são semelhantes aos de Bauman et al. (1999), em que a aplicação de rBST para vacas leiteiras, principalmente quando iniciada aos 60 dias pós-parto, apresenta marcado efeito sobre a curva de lactação, aumentando a produção de leite e a persistência de lactação.

Huber et al. (1997), Dunlap et al. (2000) e LunaDominguez et al. (2000) também avaliaram o efeito da aplicação de rBST sobre a curva de lactação e verificaram que o pico de lactação foi aumentado e estendido por maior períodos de tempo, resultando em maiores persistência da lactação e da produção de leite. Bauman (1992) observou efeito da aplicação de rBST sobre a curva de lactação de vacas Holandesas.

Na Fig. 1, mostra-se o efeito dos tratamentos sobre a produção de gordura (\% e $\mathrm{kg} / \mathrm{dia})$, em razão dos dias de lactação. A porcentagem de gordura, independentemente do tratamento, variou conforme a curva de lactação, isto é, aumentou em função dos dias de lactação, sem efeito dos tratamentos (Tab. 2). A produção de gordura no leite $(\mathrm{kg} / \mathrm{dia})$, resultado da produção de leite multiplicada pelo seu respectivo teor de gordura em porcentagem, apresentou comportamento semelhante ao da PL.

Segundo Bauman et al. (1985), o rBST pode modificar a partição dos nutrientes entre os tecidos, aumentando a eficiência produtiva das vacas leiteiras. O rBST ocasiona diversos efeitos biológicos em vários tecidos que estão envolvidos em priorizar a utilização de nutrientes para suportar incremento na síntese de leite (Bauman, 1992).

Em relação ao desempenho reprodutivo, não foram observadas diferenças $(\mathrm{P}<0,05)$ no intervalo parto-concepção e no número de serviços por concepção com a administração de rBST aos 60 e 100 dias de lactação. As taxas de gestação até os 150 dias de lactação não foram diferentes pelo teste de qui-quadrado.

Os coeficientes de variação e as médias do intervalo parto-concepção (dias), do número de serviços por concepção e da taxa de gestação (\%), de acordo com os tratamentos e até os 150 dias de lactação, são encontrados na Tab. 4. Os coeficientes de variação obtidos para o intervalo parto-concepção e o número de serviços por concepção foram altos, demonstrando grande variação nas mensurações dessas características.

Tabela 4. Médias e coeficientes de variação (CV) do intervalo parto-concepção (IPC), número de serviços por concepção (NSC), e taxa de gestação (TG), segundo os tratamentos

\begin{tabular}{lccc}
\hline Tratamento & $\begin{array}{c}\text { IPC } \\
\text { (dias) }\end{array}$ & NSC & $\begin{array}{c}\text { TG } \\
(\%)\end{array}$ \\
\hline Controle & 71,5 & 1,36 & 73,33 \\
rBST-60 & 64,9 & 2,10 & 66,67 \\
rBST-100 & 93,1 & 1,75 & 72,73 \\
CV (\%) & 46,76 & 46,82 & - \\
\hline
\end{tabular}

Bauman et al. (1985) demonstraram que vacas tratadas com rBST, quando comparadas com vacas-controle e, ainda, com vacas contemporâneas de rebanho, não apresentavam 
diferenças no intervalo parto-concepção, número de serviços por concepção e taxa de gestação. Oldenbroek et al. (1993) não verificaram diferenças na fertilidade das vacas do experimento e encontraram 88 dias de intervalo parto-concepção, tanto para vacas tratadas com rBST quanto para as controle. Segundo o autor, o número de serviços por concepção foi 1,48 e 1,49 , na ordem citada acima.

Luna-Dominguez et al. (2000) encontraram valores semelhantes para intervalo partoconcepção (105,3 e 108,3 dias) e número de serviços por concepção $(2,0$ e 2,1$)$ para vacas tratadas com rBST e controle, respectivamente. Zhao et al. (1992) e Santos et al. (2000) não verificaram efeito da administração de rBST sobre o desempenho reprodutivo, e Cole et al. (1991) também não encontraram diferenças entre os animais tratados ou não com rBST em relação à taxa de gestação (307 vacas) e ao número de serviços por concepção (239 vacas).

Lucy (2000) citou que não existem efeitos negativos inerentes à aplicação do rBST na reprodução. Esta é mais influenciada pelo aumento da produção de leite e do balanço energético negativo pós-parto. Dessa forma, quando vacas leiteiras são submetidas ao tratamento com rBST, espera-se máxima resposta produtiva e nenhuma alteração nos índices reprodutivos. As condições para a obtenção desses resultados dependem da habilidade dos especialistas em avaliar o estado nutricional e alimentar dos animais, para que seja proporcionado consumo adequado de nutrientes que sustentem as atividades produtiva $\mathrm{e}$ reprodutiva (Cole e Lucy, 1997; Lucy, 2001).

$\mathrm{Na}$ Fig. 2, encontram-se as médias do peso corporal das vacas em função dos dias de lactação. Para os três tratamentos, com o avançar da lactação, as vacas ganharam peso. Na Tab. 5, são apresentadas as equações de regressão do peso corporal, com seus respectivos coeficientes de determinação, para os tratamentos avaliados ao longo de todo período experimental.

O peso corporal aumentou linearmente em função dos dias de lactação para todos os tratamentos. Para os tratamentos-controle, rBST60 e rBST-100, a cada dia de lactação, o peso corporal foi acrescido de 315, 357 e 101g, respectivamente. O tratamento rBST-100 apresentou ganho de peso menor que os demais tratamentos, no entanto sua análise de regressão apresentou baixo coeficiente de determinação. Talvez o menor número de animais nesse tratamento, em razão do descarte de quatro vacas no início do experimento, por problemas de saúde, pode ter influenciado o resultado.

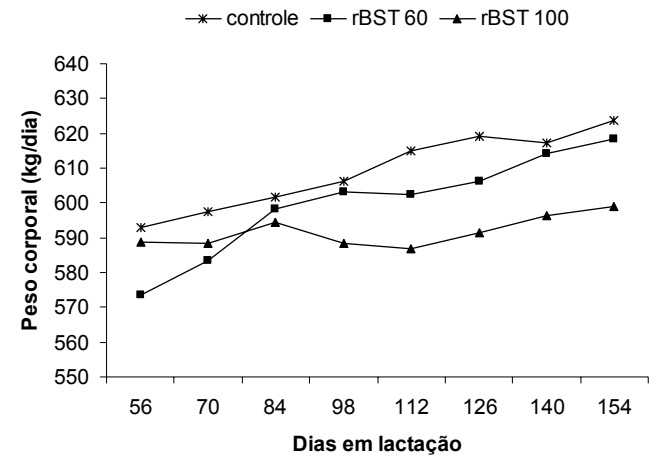

Figura 2. Peso corporal $(\mathrm{kg})$ em função dos dias de lactação, segundo os tratamentos.

Tabela 5. Equações de regressão (ER) e coeficientes de determinação $\left(\mathrm{R}^{2}\right)$ para peso corporal do $56^{\circ}$ ao $154^{\circ}$ dia de lactação, segundo os tratamentos

\begin{tabular}{lcc}
\hline Tratamento & ER & $\mathrm{R}^{2}$ \\
\hline Controle & $\hat{Y}=576,27+0,3145 \mathrm{D}$ & 94 \\
rBST-60 & $\hat{Y}=563,76+0,3568 \mathrm{D}$ & 90 \\
rBST-100 & $\hat{Y}=580,87+0,1007 \mathrm{D}$ & 43 \\
\hline
\end{tabular}

O ganho de peso semelhante, em função dos dias em lactação, para os tratamentos-controle e rBST-60, sugerem que não houve perda de peso com a utilização do rBST. Dessa forma, possivelmente pela semelhança no balanço energético das vacas nos tratamentos avaliados, não foi observado qualquer efeito negativo da aplicação do rBST sobre as condições gerais dos animais. Segundo Cole e Lucy (1997), vacas leiteiras tratadas ou não com rBST, quando do aumento na PL, apresentam balanço de energia negativo, refletido em perda de peso corporal. Entretanto, segundo esses autores, vacas leiteiras manejadas para o máximo consumo de alimentos e energia podem apresentar balanço positivo de energia, refletindo em aumento do peso corporal. 
Tarazon-Herrera et al. (2000) não encontraram efeito da aplicação de 500mg de rBST, a cada 14 dias, em relação ao controle, em 292 dias de lactação. Huber et al. (1997) verificaram aumento de peso durante o experimento e confirmaram balanço positivo de energia para animais tratados e não-tratados, com o maior ganho de peso não sendo atribuído ao aumento da camada de gordura subcutânea, pois o escore da condição corporal não foi diferente entre os tratamentos. Gibson et al. (1992) e Zhao et al. (1992) também observaram que a aplicação de rBST não afetou o ganho de peso das vacas em relação aos animais-controle, mesmo quando avaliados ao longo de toda lactação.

\section{CONCLUSÕES}

A utilização da rBST para vacas leiteiras aumentou a produção de leite, a produção de leite corrigida a $4 \%$ de gordura e a persistência de lactação, quando administrada aos 60 dias pós-parto. A rBST não tem efeito sobre a porcentagem e produção de gordura do leite. A aplicação de rBST aos 60 e 100 dias de lactação não influencia o intervalo parto-concepção, o número de serviços por concepção e a taxa de gestação aos 150 dias de lactação, bem como não afeta o ganho de peso dos animais durante o período avaliado.

\section{REFERÊNCIAS BIBLIOGRÁFICAS}

BARBANO, D.J.; LYNCH, J.M.; BAUMAN, D.E. et al. Effect of prolonged-release formulation of N-methionyl bovine somatotropina (sometribove) on milk composition. J. Dairy Sci., v.75, p.1775-1793, 1992.

BAUMAN, D.E. Bovine somatotropin: review of an emerging animal technology. J. Dairy Sci., v.75, p.3432-3451,1992.

BAUMAN, D.E.; EPPARD, P.J.; De GEETER, M.J. et al. Responses of high producing dairy cows to long-term treatment with pituitary somatotropin and recombinant somatotropin. $J$. Dairy Sci., v.68, p.1352-1362, 1985.

BAUMAN, D.E.; EVERETT, R.W.; WEILAND, W.H. et al. Production responses to bovine somatotropin in Northeast dairy herds. In:
CORNELL NUTRITION CONFERENCE FOR FEED MANUFACTURERS, 1999, Rochester. Anais... Rochester, 1999. p.109-117.

BURTON, J.H.; MacLEOD, G.K.; McBRIDE, B.W. et al. Overall efficacy of chronically administered recombinant bovine somatotropin to lactating dairy cows. J. Dairy Sci., v.73, p.2157-2167, 1990.

COLE, W.J.; LUCY, M.C. Management of reproduction in dairy herds utilizing bovine somatotropin. In: YOUNGQUIST, R.S. (Ed.). Current therapy in large animal theriogenology. Philadelphia: W.B. Saunders, 1997. p.473-478.

COLE, W.J.; MADSEN, K.S.; HINTZ, R.L. et al. Effects of recombinantly derived bovine somatotropin on reprodutive performance of dairy cattle. Theriogenology, v.36, p.573-595, 1991.

DUNLAP, T.F.; KOHN, R.A.; DAHL, G.E. et al. The impact of somatotropin, milking frequency, and photoperiod on dairy farm nutrient flows. J. Dairy Sci., v.83, p.968-976, 2000.

EPPARD, P.J.; BAUMAN, D.E.; BITMAN, D.J. et al. Effect of dose of bovine growth hormone on milk composition: a-lactalbumin, fatty acids and mineral elements. J. Dairy Sci., v.68, p.3047-3053, 1985 .

ETHERTON, T.D.; BAUMAN, D.E. Biology of somatotropin in growth and lactation of domestic animals. Physiol. Rev., v.78, p.745-761, 1998.

GIBSON, J.P.; McBRIDE, B.W.; BURTON, J.H. et al. Effects on production traits of somatotropin for up to three consecutive lactations. J. Dairy Sci., v.75, p.837-846, 1992.

HUBER, J.T.; WU. Z.; FONTES Jr., C. et al. Administration of recombinant bovine somatotropin to dairy cows for four consecutive lactations. J. Dairy Sci., v.80, p.2355-2360, 1997.

LAURENT, F.; VIGNON, B.; COOMANS, D. et al. Influence of bovine somatotropin on the composition and manufacturing of milk. J. Dairy Sci., v.75, p.2226-2234, 1992.

LUCCI, C.S.; RODRIGUES, P.H.M.; SANTOS Jr., E.J. et al. Emprego da somatotropina bovina (BST) em vacas de alta produção. Braz. J. Vet. Res. Anim. Sci., v.35, p.46-55, 1998. 
LUCY, M.C. Regulation of ovarian follicular growth by somatotropin and insulin-like growth factors in cattle. J. Dairy Sci., v.83, p.1635-1647, 2000 .

LUCY, M.C. Reprodutive loss in high producing dairy cattle: where will it end? J. Dairy Sci., v.84, p.1277-1293, 2001.

LUNA-DOMINGUEZ, J.E.; ENNS, R.M.; ARMSTRONG, D.V. et al. Reprodutive performance of Holstein cows receiving somatotropin. J. Dairy Sci., v.83, p.1451-1455, 2000 .

MATTOS, W.S. Somatotropina na pecuária de leite e de corte. In: REUNIÃO ANUAL DA SOCIEDADE BRASILEIRA DE ZOOTECNIA, 35., 1998, Botucatu. Anais... Botucatu:SBZ, 1998. p.35-52.

METABOLIC modifiers: effects on the nutrient requirements of food-producing animals. Washington, DC: National Academic, 1994.

NUTRIENT requirements of dairy cattle. 6.ed. Washinton, DC: National Academic, 1989. 158p.

NUTRIENT requirements of dairy cattle. 7.ed. Washinton, DC: National Academic, 2001. 381p.

OLDENBROEK, J.K.; GARSEN, G.J.; JONKER, L.J. et al. Effects of treatment of dairy cows with recombinant bovine somatotropin over three or four lactations. J. Dairy Sci., v.76, p.453-467, 1993.

PEEL, C.J.; BAUMAN, D.E. Somatotropin and lactation. J. Dairy Sci., v.70, p.474-486, 1987.
PEEL, C.J.; FRONK, T.J.; BAUMAN, D.E. et al. Effect of exogenous growth hormone in early and late lactation on lactational performance of dairy cows. J. Dairy Sci., v.66, p.776-782, 1983.

RICHARD, A.L.; McCUTCHEON, S.N.; BAUMAN, D.E. Responses of dairy cows to exogenous bovine growth hormone administered during early lactation. J. Dairy Sci, v. 68, p. 2385-2389, 1985.

SANTOS, J.E.P.; HUBER, J.T.; THEURER, C.B. et al. Effects of grain processing and bovine somatotropin on metabolism and ovarian activity of dairy cows during early lactation. J. Dairy Sci., v.83, p.1004-1015, 2000.

SILVA, D.J. Análise de alimentos: métodos químicos e biológicos. 2.ed. Viçosa: Imprensa Universitária/UFV, 1990. 165p.

SISTEMA de análises estatísticas e genéticas SAEG. Viçosa: UFV, 1999.

TARAZON-HERRERA, M.A.; HUBER, J.T.; SANTOS, J.E.P. et al. Effects of bovine somatotropin on milk yield and composition in Holstein cows in avanced lactation fed low or high energy diets. J. Dairy Sci., v.83, p.430-434, 2000 .

ZHAO, X.; BURTON, J.H.; McBRIDE, B.W. Lactation, health, and reproduction of dairy cows receiving daily injectable or sustained release somatotropin. J. Dairy Sci., v.75, p.3122-3130, 1992. 